\title{
Optical-flow-based motion compensation algorithm in thermoelastic stress analysis using single-infrared video
}

\author{
Tommaso Tocci $^{1}$, Lorenzo Capponi ${ }^{1}$, Roberto Marsili ${ }^{1}$, Gianluca Rossi ${ }^{1}$ \\ ${ }^{1}$ Department of Engineering, University of Perugia, via G. Duranti 93, 06125 Perugia, Italy
}

\begin{abstract}
Thermoelastic stress analysis (TSA) is a non-contact measurement technique for stress distribution evaluation. A common issue related to this technique is the rigid-displacement of the specimen during the test phase, that can compromise the reliability of the measurement. For this purpose, several motion compensation techniques have been implemented over the years, but none of them is provided through a single measurement and a single sample surface conditioning. Due to this, a motion compensation technique based on Optical-Flow has been implemented, which greatly increases the strength and the effectiveness of the methodology through a single measurement and single specimen preparation. The proposed approach is based on measuring the displacement field of the specimen directly from the thermal video, through optical flow. This displacement field is then used to compensate for the specimen's displacement on the infrared video, which will then be used for thermoelastic stress analysis. Firstly, the algorithm was validated by a comparison with synthetic videos, created ad hoc, and the quality of the motion compensation approach was evaluated on video acquired in the visible range. The research moved into infrared acquisitions, where the application of TSA gave reliable and accurate results. Finally, the quality of the stress map obtained was verified by comparison with a numerical model.
\end{abstract}

\section{Section: RESEARCH PAPER}

Keywords: Thermoelastic stress analysis; optical flow; motion compensation; mechanical stress; experimental mechanics

Citation: Tommaso Tocci, Lorenzo Capponi, Roberto Marsili, Gianluca Rossi, Optical-flow-based motion compensation algorithm in thermoelastic stress analysis using single-infrared video, Acta IMEKO, vol. 10, no. 4, article 27, December 2021, identifier: IMEKO-ACTA-10 (2021)-04-27

Section Editor: Roberto Montanini, Università di Messina and Alfredo Cigada, Politecnico di Milano, Italy

Received July 30, 2021; In final form December 9, 2021; Published December 2021

Copyright: This is an open-access article distributed under the terms of the Creative Commons Attribution 3.0 License, which permits unrestricted use, distribution, and reproduction in any medium, provided the original author and source are credited.

Corresponding author: Tommaso Tocci, e-mail: tommaso.tocci@outlook.it

\section{INTRODUCTION}

The Thermoelastic Stress Analysis (TSA) [1]-[4] is an infrared image-based technique for non-contact measurement of stress fields. This technique is based on the detection and analysis of the temperature fluctuations amplitude on the surface of a mechanical component dynamically loaded, performed by a thermal camera. Since the temperature fluctuation produced by the load are very small, a Lock-In [5], [6] data processing is normally applied to the thermal video [7], which makes it possible to emphasise phenomena at particular loading frequencies, reducing the noise effects, normally higher than thermal fluctuation induced by stress. The TSA is largely applied for many mechanical stress analysis application, FEM validation, design comparison and very useful for high resolution stress concentration analysis, non-contact stress detection in application where classical sensors have problems or limitations [8]-[11]. For an ideal application of this technique, the specimen surface should not move or make small movements throughout the test [2]. Clearly, this ideal condition is not always achievable since, for example, in the case of a specimen with low stiffness, the phenomena of deformation and displacement are very large [3]. This phenomenon is emphasised by the fact that the TSA requires the application of dynamic loads, with sufficiently high stress levels, in order to generate, by the thermoelastic principle, sufficiently high temperature fluctuations. The main problem caused by specimen movement is that, in a sequence of consecutive frames, the same pixel does not always correspond to the same part of the specimen as it moves. This phenomenon affects the lock-in operation, causing alterations in the measured stress field. Especially on the border of mechanical component, it may also happen that a pixel corresponds at a surface point or a background point alternatively: this phenomenon is commonly known as edge effect [1]. Therefore, in the thermal video, it is necessary to compensate in a certain way these movements and deformations by means of algorithms commonly called motion compensation [2]. 
The State of the Art relies on Sakagami et al. in [12], where the compensation of the motion using displacement fields is obtained with the Digital Image Correlation (DIC) [13], [14] on a second video recorded simultaneously in the visible range. The motion compensation is then applied to thermal video. This technique requires the employment of two different cameras and the double surface conditioning of the specimen: the application of speckle for the DIC and a high emissivity black paint for TSA. Silva et al. [15] simplified the compensation procedure limiting the use of a single camera both for the acquisition of the video for the DIC and the TSA techniques. In this case, however, in order to be able to make displacement measurements with the DIC, the speckle must be made through a paint with an emissivity that can be detected by an infrared camera. Nevertheless, two surface conditioning of the specimen surface are still required. Compared with Sakagami et al. [12], since one camera is employed for the two acquisitions, the alignment problems, which are very evident in the use of two cameras that should ideally be positioned in the same place, are greatly reduced. This research proposes an algorithm that allows the compensation of the rigid motion using a single thermal video with a single step specimen preparation, from which it will be possible to perform both the compensation and the TSA.

Therefore, compared to the state of the art, it will no longer be necessary to first prepare the specimen for measurement with DIC, then remove the speckle and finally prepare the surface for analysis by the TSA, but it will be sufficient to do only the preparation for the TSA analysis, significantly reducing the time required for the preparation phase. In addition, with a single thermal video it is possible to obtain both the motion compensation field and the stress field, thus also reducing the time of the experimental phase. This technique allows to carry out tests in which it is not possible to repeat the measurement, such as, for example, structural failure or random tests. The motion compensation phase sees the replacement of the DIC with a computer vision technique called Optical-Flow [16]-[18], which is based on motion of visual-features, such as corners, edges, ridges and textures in two consecutive frames of a video scene [19], [20]. Within optical-flow methods, the differentiation between sparse and dense approach is fundamental [18]. There are many different types of algorithms [21] based on different correlation techniques, such as gradient-based, based on the brightness constancy equation [22] or region-based approaches rely on the correlation of different features like normalized crosscorrelation and Laplacian correlation [23], [24]. Optical-Flow has been widely applied to measure displacement fields in solid bodies [25]-[27]. In this work, the dense Optical-Flow Farneback algorithm was implemented [28], [29].

A Nylon specimen, realized by additive manufacturing, was tested applying a sinusoidal load along its y-axis, so that the movement of the specimen is exclusively along the vertical direction. In a preliminary step the algorithm was validated by analysing synthetically generated videos with imposed motion. The coincidence between the displacement measured by the implemented algorithm and the displacement imposed on the synthetic video was then evaluated. The algorithm was then tested in visible video to evaluate the efficiency of the motion compensation and then it is applied to a thermal video for the TSA.

The manuscript is organized as follows: in Sec. 2, the implemented algorithm, the test bench used and the experimental setup are presented. In Sec.3 the algorithm is validated and the results discussed. Sec. 4 draws the conclusions.

\section{MATERIALS AND METHOD}

\subsection{Algorithm Implementation}

All the algorithms of this work have been implemented in a Python environment. Below is the description of the two main algorithms: one for measuring the displacement field and one for compensating for motion the thermal film.

\subsubsection{Algorithm for displacement field measurement}

Data processing for the purpose of determining the displacement field is not carried out on the original video matrix but on a copy of it. Especially for thermal video, the copy is normalised from the 14-bit of the original video to 8-bit. The compensation field detected by the 8-bit video is then applied to the original 14-bit video. Each pair of frames, before being calculated by Optical-Flow, is subjected to a pre-processing phase, designed to reduce noise (very evident especially in infrared images) and to make a masking of the sample, which will be used later. Each acquired frame is filtered, in sequence, through a Gaussian filter [30], [31] with 5x5 kernel, a morphological transformation of dilation and erosion [30]-[32] , with $3 \times 3$ kernel. This produces a good noise reduction, especially in the thermal image, as shown in Figure 1.

The result obtained is then sorted through an image binarization algorithm [30], which returns a black and white binary field, showing the non-presence/presence of the specimen in a given pixel. Example of mask of the specimen are shown in Figure 2. As can be seen in the figure, the mask quality of the visible image is higher than that of the infrared image, but in both cases a good result is obtained.

The Farneback Optical-Flow algorithm [28], [29] is now applied to each pair of frame. Each frame of the video is compared with a reference frame, which is usually the first frame of the video. The displacement field measured in this way indicates the displacement of the $i$-th frame with respect to the reference one. The displacement field indicates the displacement

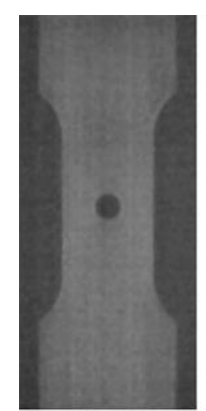

(a)

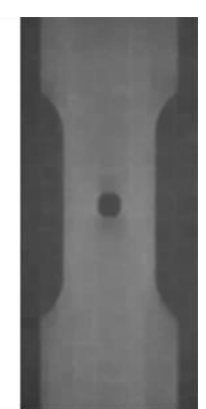

(b)
Figure 1. Image pre-processing: (a) Original frame (b) Filtered frame.

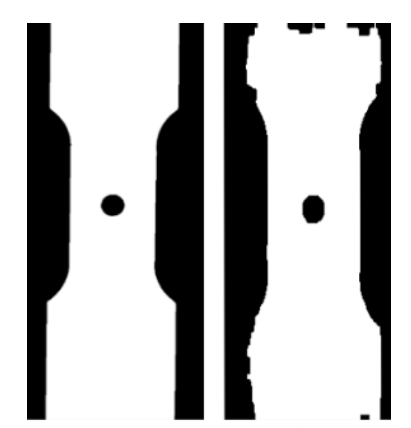

(a)

(b)

Figure 2. Specimen mask: (a) Visible video (b) Infrared video. 


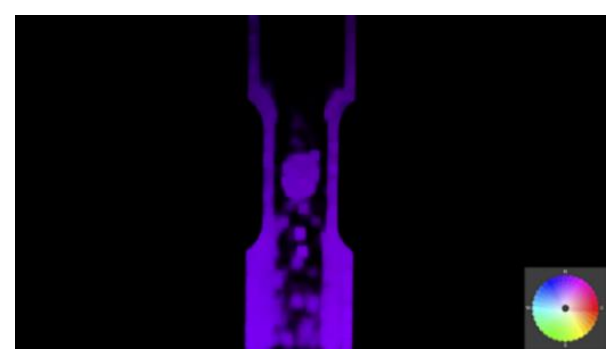

Figure 3. Example of rough displacement field.

undergone by each pixel between the two frames. The field is displayed in HSV colour code, where the colour indicates the direction of displacement and the colour intensity indicates the magnitude. An example of a rough displacement field is given in Figure 3: it can be seen that the displacement is mainly traced along the edges of the specimen and that the inner areas are detected as stationary. This requires a phase of improvement of the displacement field.

We start with an initial masking, which eliminates all displacement vectors detected outside the specimen due to noise. Operationally, the displacement matrix is multiplied by the mask matrix, as shown in Figure 4.

Since it was decided to compensate for motion only along the vertical axis, the maximum displacement detected for each line is determined, which is likely to be located along the edges of the specimen. At this point, each row of the matrix has a constant value over the length of the frame, ideally including areas outside the specimen, as shown in Figure 5.

A new masking is then performed. To better understand this, it can be said that the first masking is intended to prevent the algorithm from taking for granted a maximum displacement that could be detected at a point outside the specimen, while the
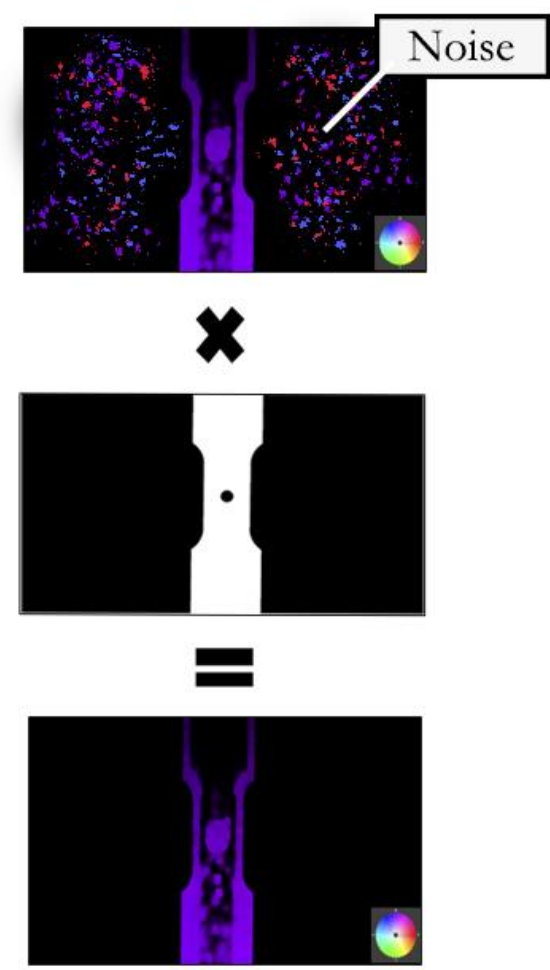

Figure 4. First masking operation: incorrect displacement vectors are masked out.

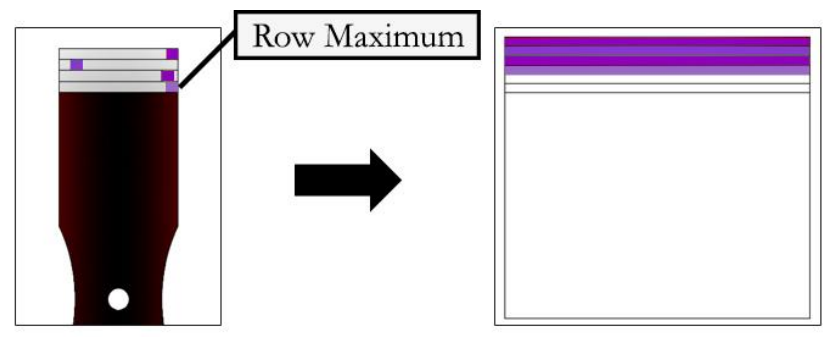

Figure 5. Calculation of maximum displacement line by line.
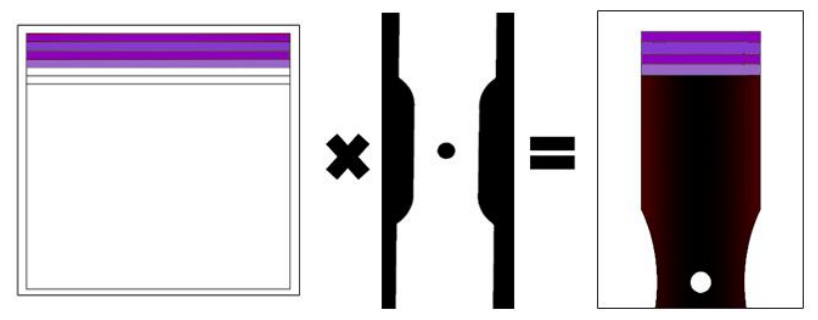

Figure 6. Second masking operation: compensation field is created.

second is applied to eliminate line by line displacement values per pixel outside the geometry of the specimen.

Finally, the field of compensated displacements is filtered with a blur filter, in order to make the field of compensated displacements uniform in pixels, as shown in Figure 7. Compared to the one shown in Figure 3, a greater uniformity and regularity can be observed.

\subsubsection{Algorithm for motion compensation}

Motion compensation takes place on the original visible video or the original 14-bit infrared video, which has not been altered in any way during the previous step. A column vector containing the displacement of each row is then extracted from the displacement field. This displacement vector, once inverted, represents the motion to be applied to each row of the original video for motion compensation. In order to improve the compensation and make it smoother and more consistent, this compensation vector is manipulated using the Kalman filter [33], as shown in Figure 8. This vector is called Compensation Vector.

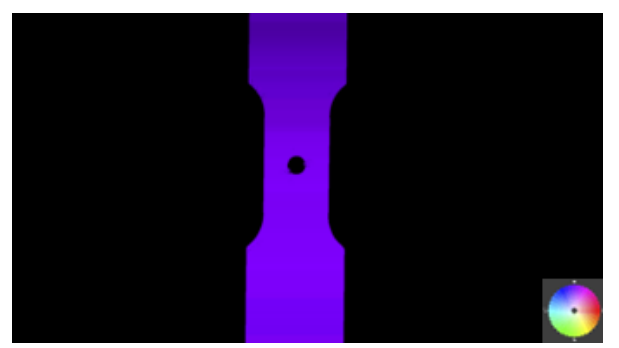

Figure 7. Example of smooth and masked displacement field.

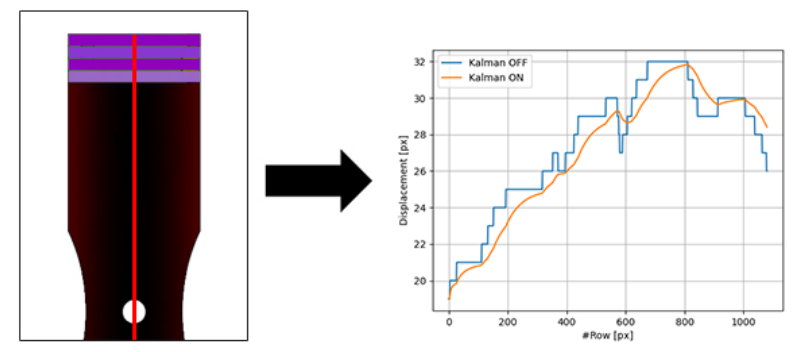

Figure 8. Extraction and smoothing of the compensation vector. 


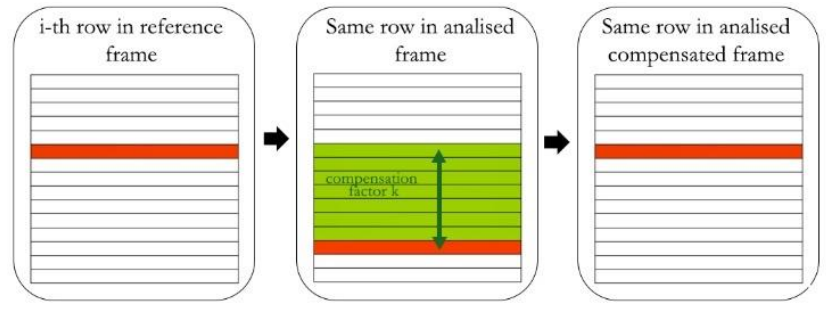

Figure 9. Motion compensation algorithm.

We then move on to the actual compensation by applying a shift to the lines of the video to be compensated using the information contained in the compensation vector. The $i$-th row of the original video is moved to position $i+k$, where $k$ is the value of the compensation move, as shown in Figure 9. A check is also made to see if this move would place the line outside the maximum frame size, in which case no compensation is done for that line. The output is a compensated matrix starting from the original video, which contains the thermal and stress information, but which is not manipulated in any way: it is only compensated by moving the position of the lines, but not by modifying their content in any way.

\subsection{Test bench and acquisition system}

The test bench used during the experimental campaign consists of a shaker Sentek L1024M, over which a structure for tensile testing was built, as shown in Figure 10.

A specimen with identical geometry to that used by Sakagami et al. [12] was used. The specimen was produced using FDM printing in Nylon 6-6 with 100\% infill ratio and a layer height of $0.1 \mathrm{~mm}$. The material has a tensile strength of $80 \mathrm{MPa}$ and an elastic module of 2.2 GPa. Two pairs of clamps, one connected to the head of the shaker and the other to the fixed part of the structure, were used to fix the specimen, as shown in Figure 11 A load cell is positioned at the top of the specimen in order to monitor the loads exchanged during testing.

Two cameras were used: one for visible video and one for infrared video. A Canon EOS 7D reflex camera with $24 \mathrm{~mm}-$ $70 \mathrm{~mm}$ lens capable of acquiring 1920x1080 pixels resolution video at $30 \mathrm{fps}$ was used. Therefore, a FLIR A6751sc thermal camera with a cooled sensor was used for infrared video acquisition. This thermal camera captures $640 \times 512$ pixels resolution video at a framerate of $100 \mathrm{fps}$ and has a thermal

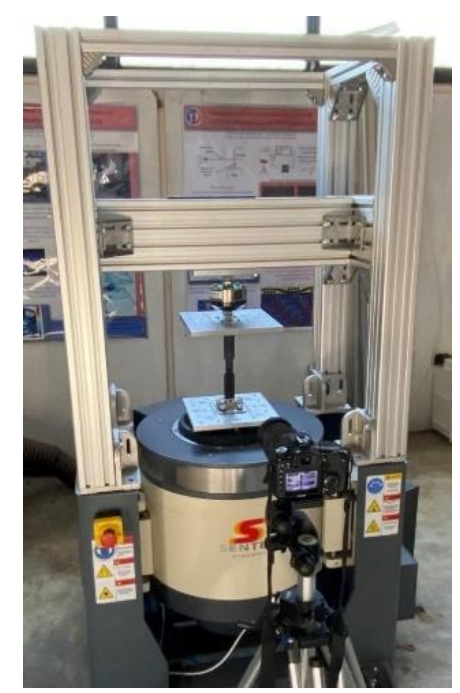

Figure 10. Test Bench.

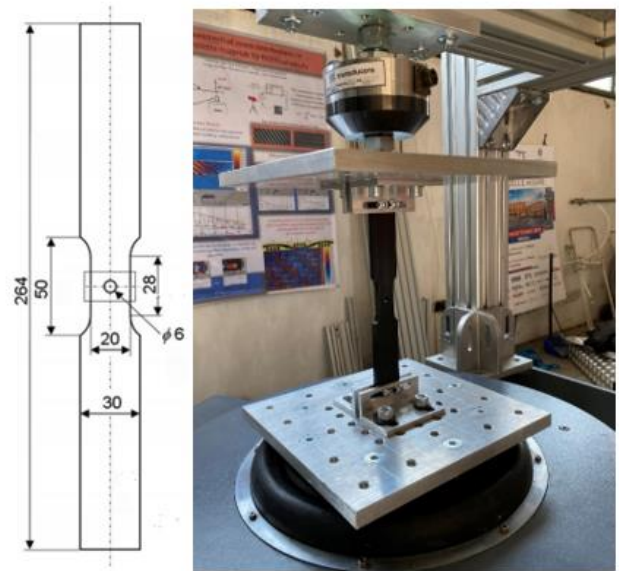

Figure 11. Specimen geometry and grasping.

sensitivity of less than $20 \mathrm{mK}$. Both video cameras were positioned in front of the specimen at the height of the specimen hole.

\subsection{Experimental campaign}

The experimental campaign was divided into two steps: first, videos were acquired in the visible at different frequencies in order to test and validate the compensation algorithm. Subsequently, infrared videos were acquired at different frequencies and different load levels in order to test the effectiveness of the motion compensation algorithm for thermoelastic application. A summary is given in Table 1 .

\section{RESULTS}

\subsection{Algorithm validation}

Since a validation phase of the motion compensation algorithm described above is required, it was decided to generate synthetic videos in which a body is present that is clearly identifiable from the Optical-Flow and has a known imposed displacement. The motion of the body in the synthetic video is imposed in a sinusoidal regime with imposed amplitude and frequency. Several videos have been generated with different

Table 1. Summary of the experimental campaign.

\begin{tabular}{cccc}
\hline Frequency in $\mathrm{Hz}$ & Sinusoidal Load in $\mathrm{N}$ & Visible & Infrared \\
\hline \multirow{2}{*}{4} & 1500 & & $\mathrm{X}$ \\
& 3000 & $\mathrm{X}$ & $\mathrm{X}$ \\
\multirow{2}{*}{5} & 1500 & & $\mathrm{X}$ \\
& 3000 & & $\mathrm{X}$ \\
\multirow{2}{*}{8} & 1500 & $\mathrm{X}$ & $\mathrm{X}$ \\
& 3000 & $\mathrm{X}$ \\
\hline
\end{tabular}

Table 2. Summary of synthetic video.

\begin{tabular}{ccc}
\hline \#Syntethic Video & Frequency in $\mathrm{Hz}$ & Amplitude in pixel \\
\hline 1 & 1 & 5 \\
2 & 1 & 10 \\
3 & 1 & 20 \\
4 & 4 & 5 \\
5 & 4 & 10 \\
6 & 4 & 20 \\
7 & 8 & 5 \\
8 & 8 & 10 \\
9 & 8 & 20 \\
\hline
\end{tabular}


operating conditions, as illustrated in Fehler! Verweisquelle konnte nicht gefunden werden..

In order to validate the displacement fields obtained by Optical-Flow, a comparison was made between the displacement detected in different survey lines on the object placed in the video and the displacement imposed during the generation of the synthetic video. In each video, three horizontal survey lines are defined: an upper red line, a central green line and a lower blue line. Note that the red line and the blue line run alternately from the marker area. Some results obtained at $4 \mathrm{~Hz}$ and $8 \mathrm{~Hz}$ are shown below. However, validation was carried out for each synthetic video generated, obtaining superimposable results between detected and imposed displacement. It is also reported the HSV displacement field, where it is possible to see how the detected displacement occupies the zone with a colour gradation going from green to violet, which corresponds in fact to the colour connected to displacements perfectly along the y-axis; this gives us a further confirmation of the correct application of the Optical-Flow. In Figure 12 you can see how the green line in the vicinity of the marker areas gives us a maximum displacement of about 5 pixels which is exactly coincident with the imposed one. The same result is obtained at Figure 13 where a displacement of 20 pixels is found, coinciding with the imposed one. Also, from the point of view of the frequency of the load, it can be said that this face does not have a negative influence on the measurement of the displacement.

The compensation algorithm is then validated and tested by computing visible video, which is easier to process due to the lower amount of noise than infrared video. As for the videos in the visible, the quality of motion compensation is verified by comparing the positions of the lower end of the hole in the specimen. A line is placed at the lower end of the hole, as shown in Figure 14. It can be seen from the model that in the uncompensated frame, because there is rigid motion, the position of the hole is varied. Thanks to the compensation of the

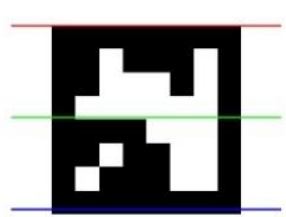

(a)
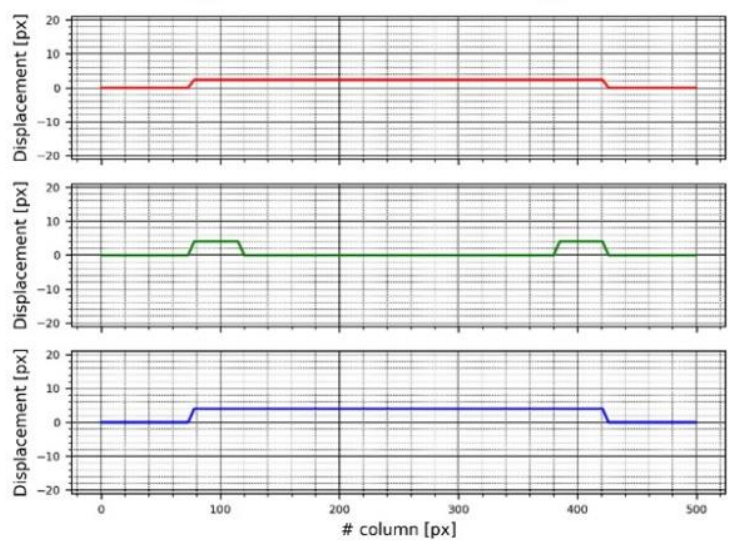

(c)

Figure 12. Synthetic video \#7: (a) Survey lines monitored (b) Field of measured displacement (c) Dynamic plot of measured displacement along survey lines.

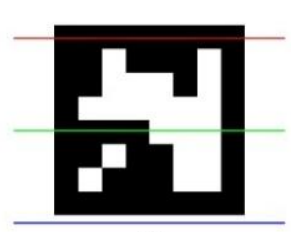

(a)

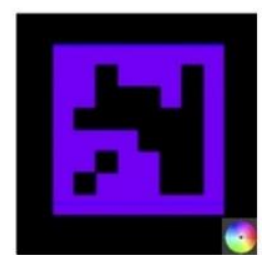

(b)
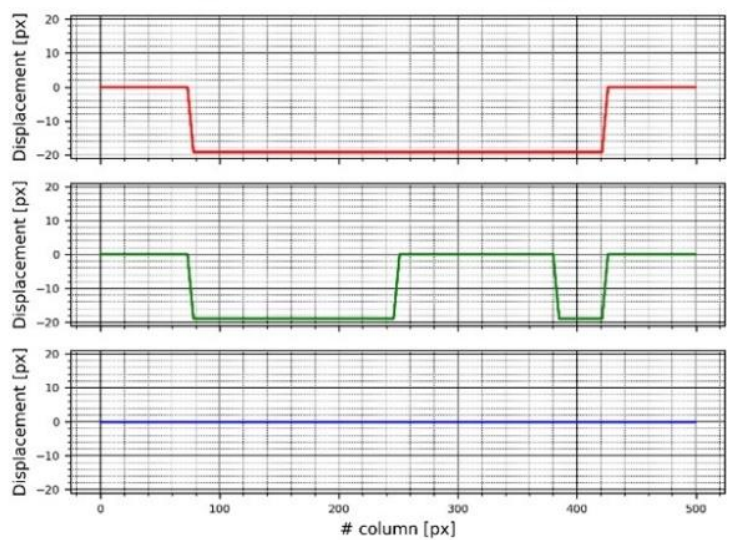

(c)

Figure 13. Synthetic video \#6: (a) Survey lines monitored (b) Field of measured displacement (c) Dynamic plot of measured displacement along survey lines.

motion instead, the position of the centre of the hole of the $i$-th frame, is brought back to the position of the reference frame.

To illustrate the result obtained by means of compensation, we will insert the superimposed image of the 3 frames, as shown in Figure 15: reference, uncompensated frame and compensated frame; it is clearly evident that in the compensated case, the position of the hole is in line with that of the reference frame.

We also report the overlapped frames between the reference image and the uncompensated one (Figure 16-a) and between the reference image and the compensated one (Figure 16-b). Also, in this case it is clear that in the compensated image the overlapping is almost perfect, because the edges of the reference image are not visible. On the contrary in the not compensated one we have that the displacement is well evident.

In the following, the effect of motion compensation was evaluated by measuring the displacement of the centre of the hole in relation to the reference position. It can be seen that, for a load frequency of $4 \mathrm{~Hz}$, a displacement attenuation of approx. $92 \%$ was obtained. If, on the other hand, $8 \mathrm{~Hz}$ is taken as the load frequency, since smaller amplitudes occur as the frequency increases, compensation of approx. $80 \%$ is achieved. In both cases, this is a good result. Results are shown in Table 3.

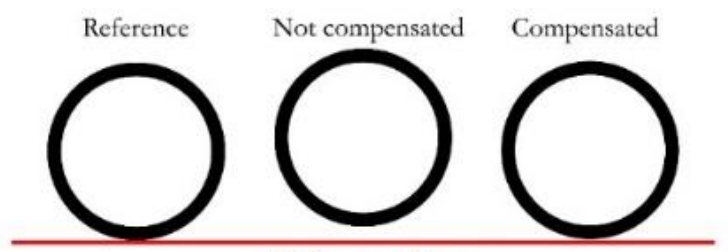

Reference Line

Figure 14. Diagram of the comparison model between the reference hole and the uncompensated/compensated one. 


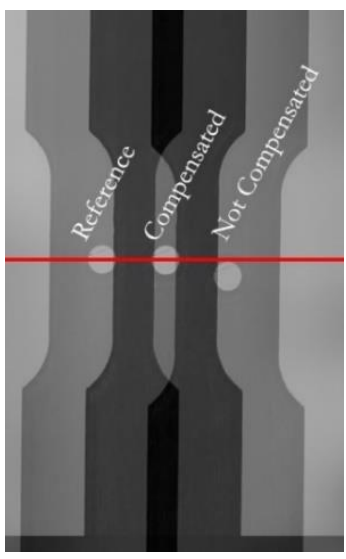

Figure 15. Comparison of the position of the 3 holes in the reference, uncompensated and compensated frame.

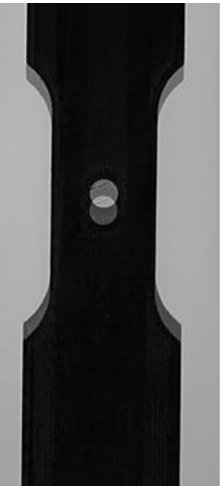

(a)

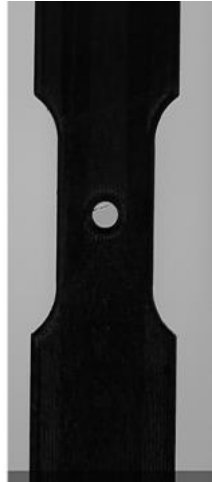

(b)
Figure 16. Frame comparisons: (a) Reference - Non-compensated (b) Reference-Compensated.

\subsection{Thermoelasticity application}

The following graphs (Figure 17, Figure 18 and Figure 19) compare the stress fields obtained from uncompensated and compensated infrared videos. The stress distribution field is expressed in terms of temperature variation in ${ }^{\circ} \mathrm{C}$.

Analysis of the fields clearly shows the improvement in quality produced by the motion compensation. In the uncompensated image, a significant component of edge effect is visible, due to the rigid motion of the specimen. This phenomenon makes it difficult to detect distress zones, especially in the areas close to the borehole, where a higher stress concentration is expected. After compensation, however, it is easiest to identify the typical stress distribution around the hole. It is also noticeable that the field is much sharper than the uncompensated one. In order to validate the result, the TSA stress fields are compared with those obtained from the FEM model. A coherent trend of the experimental fields in relation to the numerical ones can be seen.

Table 3. Measurement of hole displacement uncompensated and compensated configuration

\begin{tabular}{ccc}
\hline Test & $\begin{array}{c}\text { Not compensated } \\
\text { displacement in pixel }\end{array}$ & $\begin{array}{c}\text { Compensated } \\
\text { displacement in pixel }\end{array}$ \\
\hline $4 \mathrm{~Hz}$ - Frame 11 & 36 & 3 \\
$4 \mathrm{~Hz}-$ Frame 118 & 35 & 3 \\
$4 \mathrm{~Hz}$ - Frame 226 & 33 & 3 \\
$8 \mathrm{~Hz}$ - Frame 16 & 9 & 2 \\
$8 \mathrm{~Hz}-$ Frame 124 & 11 & 2 \\
$8 \mathrm{~Hz}$ - Frame 228 & 10 & 2 \\
\hline
\end{tabular}
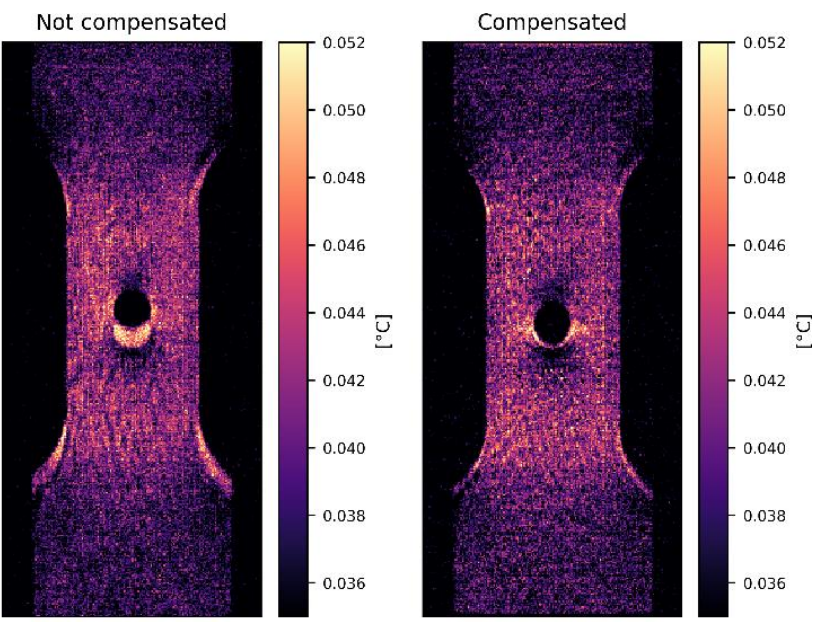

Figure 17. $4 \mathrm{~Hz}$ infrared video: comparison of distress fields of uncompensated and compensated video.
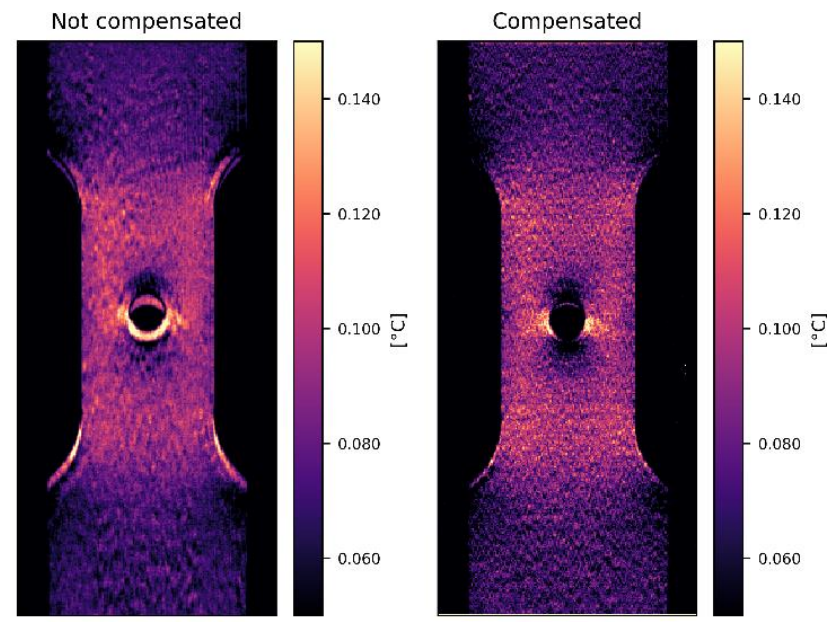

Figure 18. $5 \mathrm{~Hz}$ infrared video: comparison of distress fields of uncompensated and compensated video.
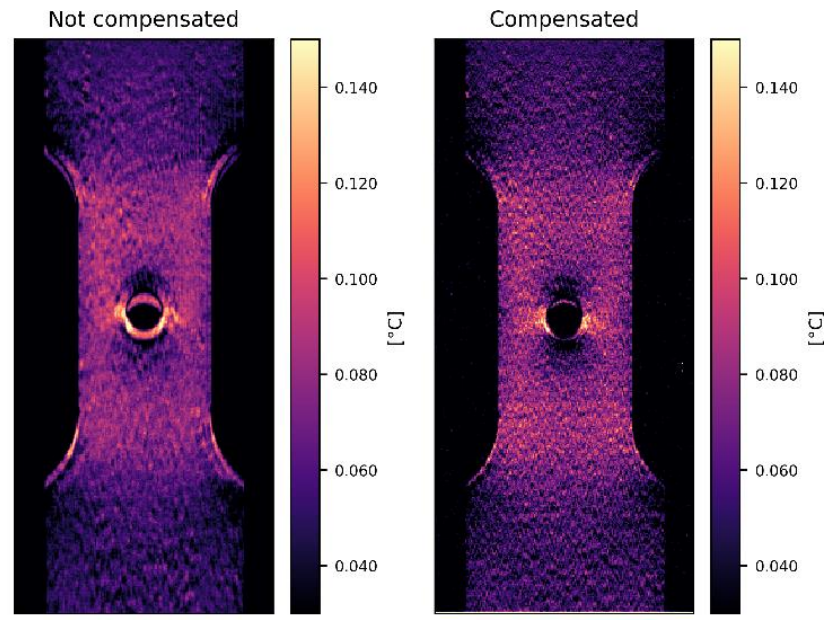

Figure 19. $8 \mathrm{~Hz}$ infrared video: comparison of distress fields of uncompensated and compensated video.

Below, in Figure 20, is the graph comparing the stressconcentration factor at the hole in the uncompensated, compensated video and the FEM model along the two check lines passing through its centre.

In order to be able to compare the experimental stress profiles with the FEM, the concentration of the normalised stresses was 

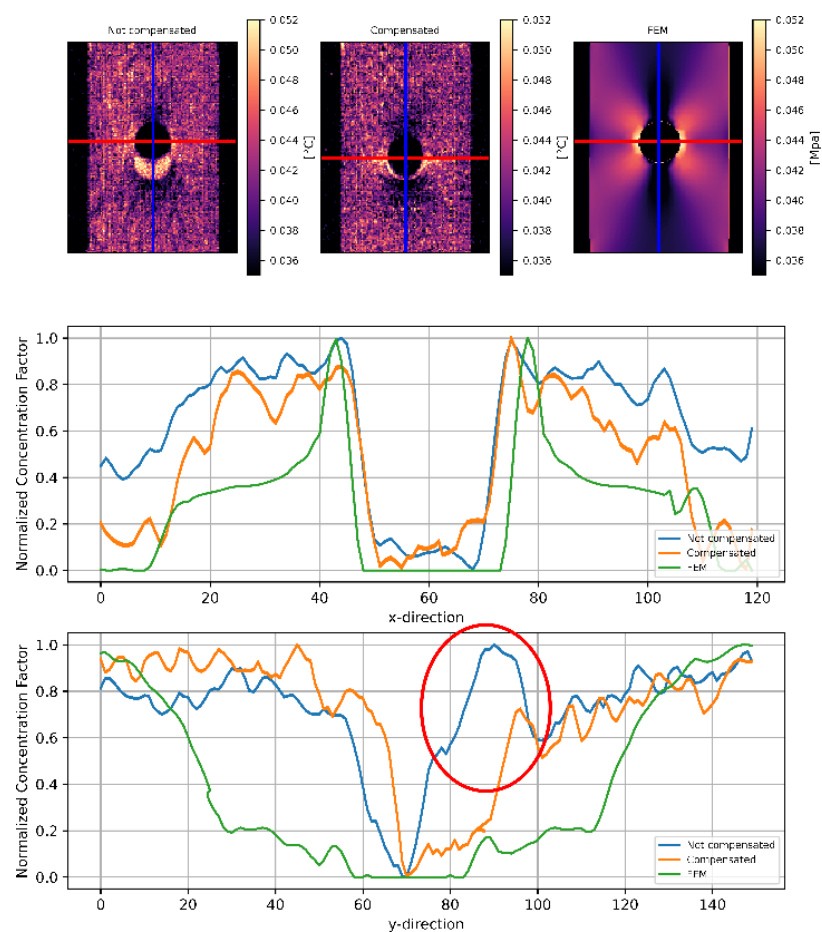

Figure 20. Comparison of stress concentration in uncompensated, compensated and FEM video along the two check lines passing through the centre of the hole.

evaluated, i.e., by dividing the series of values by the maximum of the series. It can be seen that, along the vertical direction, in the uncompensated video there is an inversion of the stress concentration at the bottom of the hole (red circle in Figure 20), which in this case corresponds to the area between 80 and 100 along the y-direction, due to the edge effect. On the contrary, this inversion of stress concentration is not present in the compensated video, which presents a trend similar to that of the FEM model.

\section{CONCLUSIONS}

The implementation of a new motion compensation algorithm for thermoelastic applications respect the state of the art was proposed in this research. The algorithm was first validated by means of a series of synthetic videos generated in such a way as to have a known imposed displacement. Then, the tests were carried out on videos in the visible range where the performances of the motion compensation were evaluated, which was around $92 \%$ for $4 \mathrm{~Hz}$ displacements and $80 \%$ for $8 \mathrm{~Hz}$ displacement. Stress distribution fields produced from the same compensated and uncompensated video were compared. The blurring and edge effects produced by the motion were almost completely eliminated, making it possible to correctly measure the stress field, especially in the area around the hole. This result was compared with stress fields obtained from finite element analysis. Videos were tested at different frequencies in order to verify the robustness of the algorithm. Finally, the normalised stress concentration was compared along two perpendicular survey lines passing through the centre of the hole. It can be seen that, along the $y$-compensation line, the inversion of tension due to the effect of motion was significatively reduced by the here proposed motion compensation algorithm.

\section{REFERENCES}

[1] W. Thomson, On the Dynamical Theory of Heat, Earth Environ. Sci. Trans. R. Soc. Edinburgh, vol. 20, no. 2, pp. 261-288, 1853.

[2] W. Weber, Über die specifische Wärme fester Körper, insbesondere der Metalle, Ann. Phys., vol. 96, no. 10, pp. 177-213, 1830 .

[3] J. M. Dulieu-Barton, S. Quinn, C. Eyre, P. R. Cunningham, Development of a temperature calibration device for thermoelastic stress analysis, in Applied Mechanics and Materials, 2004, vol. 1, pp. 197-204.

DOI: $10.4028 /$ www.scientific.net/AMM.1-2.197

[4] J. M. Dulieu-Barton, Thermoelastic stress analysis, Opt. methods solid Mech. a Full-f. approach, pp. 345-366, 2012.

[5] N. Harwood, W. M. Cummings, Applications of thermoelastic stress analysis, Strain, vol. 22, no. 1, pp. 7-12, 1986.

DOI: $10.1111 /$ j.1475-1305.1986.tb00014.x

[6] N. Harwood, W. M. Cummings, Calibration of the thermoelastic stress analysis technique under sinusoidal and random loading conditions, Strain, vol. 25, no. 3, pp. 101-108, 1989.

DOI: $10.1111 / \mathrm{j} .1475-1305.1989 . t b 00701 . \mathrm{x}$

[7] L. Capponi, LolloCappo/pyLIA: Digital lock-in analysis. Zenodo, 2020, DOI: $10.5281 /$ zenodo.4043175

[8] G. Allevi, L. Capponi, P. Castellini, P. Chiariotti, F. Docchio, F. Freni, R. Marsili, M. Martarelli, R. Montanini, S. Pasinetti, A. Quattrocchi, R. Rossetti, G. Rossi, G. Sansoni, E. P. Tomasini, Investigating additive manufactured lattice structures: a multiinstrument approach, IEEE Trans. Instrum. Meas., 2019, pp. 2459 $-2467$.

DOI: $10.1109 /$ TIM.2019.2959293

[9] R. Montanini R. Montanini, G. Rossi, A. Quattrocchi, D. Alizzio, L. Capponi, R. Marsili, A. D. Giacomo, T. Tocci, Structural Characterization of Complex Lattice Parts by Means of Optical Non-Contact Measurements, in 2020 IEEE International Instrumentation and Measurement Technology Conference (I2MTC), 2020, pp. 1-6. DOI: $10.1109 /$ I2MTC43012.2020.9128771

[10] L. Capponi, J. Slavič, G. Rossi, M. Boltežar, Thermoelasticitybased modal damage identification, Int. J. Fatigue, vol. 137, Aug. 2020, p. 105661.

DOI: $10.1016 /$ j.ijfatigue.2020.105661

[11] L. Capponi, R. Marsili, G. Rossi, T. Zara, Thermoelastic stress analysis on rotating and oscillating mechanical components, Int. J. Comput. Eng. Res., vol. 10, no. 6, 2020, pp. 2250-3005.

[12] T. Sakagami, N. Yamaguchi, S. Kubo, T. Nishimura, A new fullfield motion compensation technique for infrared stress measurement using digital image correlation, J. Strain Anal. Eng. Des., vol. 43, no. 6, 2008, pp. 539-549. DOI: $\underline{10.1243 / 03093247 \text { JSA360 }}$

[13] B. Pan, K. Qian, H. Xie, A. Asundi, Two-dimensional digital image correlation for in-plane displacement and strain measurement: a review, Meas. Sci. Technol., vol. 20, no. 6, 2009, p. 62001. DOI: $10.1088 / 0957-0233 / 20 / 6 / 062001$

[14] Jason Cantrell, Sean Rohde, David Damiani, Rishi Gurnani, Luke DiSandro, Josh Anton, Andie Young, Alex Jerez, Douglas Steinbach, Calvin Kroese, Peter Ifju, Experimental characterization of the mechanical properties of 3D printed ABS and polycarbonate parts, Conf. Proc. Soc. Exp. Mech. Ser., vol. 3, 2017, pp. 89-105.

DOI: $10.1007 / 978-3-319-41600-7 \quad 11$

[15] M. L. Silva, G. Ravichandran, Combined thermoelastic stress analysis and digital image correlation with a single infrared camera, J. Strain Anal. Eng. Des., vol. 46, no. 8, 2011, pp. 783-793. DOI: $10.1177 \% 2 F 0309324711418286$

[16] J. L. Barron, D. J. Fleet, S. S. Beauchemin, Performance of Optical-Flow techniques, Int. J. Comput. Vis., vol. 12, 1994, no. 1, pp. 43-77.

DOI: $\underline{10.1007 / \mathrm{BF} 01420984}$ 
[17] B. D. Lucas, T. Kanade, An iterative image registration technique with an application to stereo vision, 1981.

[18] B. D. Lucas, Generalized image matching by the method of differences. Phd. Carnegie Mellon University, 1984.

[19] P. Turaga, R. Chellappa, A. Veeraraghavan, Advances in videobased human activity analysis: challenges and approaches, in Advances in Computers, vol. 80, Elsevier, 2010, pp. 237-290. DOI: $10.1016 /$ S0065-2458(10)80007-5

[20] S. Akpinar, F. N. Alpaslan, Video action recognition using an Optical-Flow based representation, in Proceedings of the international conference on image processing, computer vision, and pattern recognition (IPCV), 2014, p. 1.

[21] T. Fuse, E. Shimizu, M. Tsutsumi, A comparative study on gradient-based approaches for Optical-Flow estimation, Int. Arch. Photogramm. Remote Sens., vol. 33, no. B5/1; PART 5, pp. 269_ 276, 2000.

[22] S. Baker, I. Matthews, Lucas-kanade 20 years on: A unifying framework, Int. J. Comput. Vis., vol. 56, no. 3, pp. 221-255, 2004 DOI: 10.1023/B:VISI.0000011205.11775.fd

[23] W. K. Pratt, Correlation techniques of image registration, IEEE Trans. Aerosp. Electron. Syst., no. 3, pp. 353-358, 1974

[24] P. J. Burt, Local correlation measures for motion analysis: a comparative study, 1982.

[25] G. Allevi, L. Casacanditella, L. Capponi, R. Marsili, G. Rossi, Census Transform Based Optical-Flow for Motion Detection during Different Sinusoidal Brightness Variations, in Journal of
Physics: Conference Series, 2018, vol. 1149, no. 1, p. 12032. DOI: $10.1088 / 1742-6596 / 1149 / 1 / 012032$

[26] D. Gorjup, J. Slavič, A. Babnik, M. Boltežar, Still-camera multiview Spectral Optical-Flow Imaging for 3D operatingdeflection-shape identification, Mech. Syst. Signal Process., vol. 152, p. 107456, 2021.

DOI: $10.1016 /$ i.ymssp.2020.107456

[27] J. Javh, J. Slavič, M. Boltežar, Experimental modal analysis on fullfield DSLR camera footage using spectral Optical-Flow imaging, J. Sound Vib., vol. 434, pp. 213-220, 2018. DOI: $10.1016 /$ i.jsv.2018.07.046

[28] G. Farnebäck, Polynomial expansion for orientation and motion estimation. Linköping University Electronic Press, 2002.

[29] G. Farnebäck, Two-frame motion estimation based on polynomial expansion, in Scandinavian conference on Image analysis, 2003, pp. 363-370. DOI: $10.1007 / 3-540-45103-X \quad 50$

[30] R. Szeliski, Computer vision: algorithms and applications. Springer Science \& Business Media, 2010.

[31] G. Bradski, A. Kaehler, Learning OpenCV: Computer vision with the OpenCV library. O’Reilly Media, Inc., 2008.

[32] A. Eleftheriadis, A. Jacquin, Image and Video Segmentation, in Advances in Image Communication, 1999, pp. 1-68.

[33] G. Welch, G. Bishop, An introduction to the Kalman filter, 1995. 